\title{
A COMPARATIVE STUDY - MEDICAL IMAGING TECHNIQUES: GPU-BASED OR PARALLELIZED?
}

\author{
Jasleen Kaur $^{1}$ and Anil Kumar ${ }^{2}$
}

Abstract- Medical Imaging is a way of developing visual illustrations of a human body and as a result, helps in the proper diagnosis and treatment of a particular disease. Medical imaging has proved to be a boon for medical science. Numerous approaches and algorithms are there to implement different medical imaging techniques. This paper compares the different approaches and algorithms used in the medical imaging techniques. The different techniques are compared on the basis of whether they are implemented with the help of GPU or a parallelization based technique has been used.

Keywords - Distributed Processing, GPU, Loosely Coupled, Medical Imaging techniques, Parallel Processing.

\section{INTRODUCTION}

The process as well as the technique of developing visual illustrations of a body's interior for medical treatment as well as clinical evaluation, and also for the representing visually physiology i.e. the human organs' function, is known as medical imaging. Medical imaging is helpful in revealing the internal body structures as well as in diagnosing them and the treatment of the diseases related to them. The standard which is used almost everywhere for storing, exchanging as well as transmitting medical images is DICOM (Digital Imaging and Communication in Medicine). The DICOM Standard incorporates All the necessary protocols that are made use of in imaging techniques like computed tomography (CT), radiography, ultrasonography, radiation therapy as well as magnetic resonance imaging (MRI) are incorporated in the DICOM standard may be provided to understand easily about the paper.

\subsection{Medical Imaging Techniques}

1.1.1Radiography

The imaging technique which makes use of the electromagnetic radiation; especially, X-rays is known as radiography. An X-ray generator produces a heterogenous beam of X-rays. The projection of this beam is done towards the object for the creation of required image.

\subsubsection{Computed Tomography}

The diagnostic imaging test which is used for the creation of precise illustrations or images related to body organs, soft tissue, bones, soft tissue as well as blood vessels; is known as CT or Computed Tomography. CT scanning is usually the most effective means for the detection of various cancers as these images make the confirmation of tumor very clear to the doctor and help in determining the location and size of the tumor.

\subsubsection{Magnetic Resonance Imaging(MRI)}

The test which makes use of pulses of radio wave energy and magnetic field for creating images of structures and organs within the human body is called Magnetic Resonance Imaging or precisely MRI. The pictures that are obtained from an MRI scan are in the form of digital images, which in turn makes their storage and processing easier on a computer.

\subsubsection{Ultrasonography}

Ultrasonography makes use of ultrasound waves for producing the images related to internal body organs as well as other tissues. The goal of ultrasonography is usually finding the source of the disease which the patient is suffering from. The most common form of ultrasonography is obstetric ultrasound which is widely used in the examination of pregnant women. X-rays are not used in the technique of ultrasonography.

\section{RELATED WORK}

Doel et al 2017[1] have proposed GIFT-Cloud which is a data sharing as well as collaboration platform designed for carrying out research in medical imaging. Mingliang et al 2016[2] have improved the NLM algorithm which now involves noise weighting function as well as parallelizing to denoise medical images. Guo et al 2016[3] have developed an enhanced iterative algorithm, thus combining TVM (Total variation minimization) and RSF (Region scalable fitting) model. This improved algorithm can be used for inspecting defects in large-sized objects as well as for a unilateral offcentered scanning trajectory. Nguyen et al 2016[4] have proposed the existing parallel hybrid architecture in a

\footnotetext{
${ }^{1}$ Department of CET, Guru Nanak Dev University, Amritsar-143001

2 Department of CET, Guru Nanak Dev University, Amritsar-143001
} 
distributed version for solving computation time problem. The authors have also proposed a new method for computing the coefficients of filters' by enhancing filters' parameters by considering the current voxel's neighbourhod more accurately. Bychkovsky et al 2016 [5] have surveyed imaging in early and adavanced breast cancer. The authors have discussed thoroughly the optimal use of staging imaging in both early as well as locally advanced breast cancer, surveillance imaging's role in detecting recurrent disease in Stage 0-III breast cancer as well as the authos have discussed how patients with metastatic breast cancer should be followed with advanced imaging. Feng et al 2016[6] have proposed a scalable 3-dimensional hybrid MPI+Threads parallel Delaunay image-to-mesh conversion algorithm which has its scalability on a distributed memory-clusters. Schwerter et al 2016[7] have proposed an approach for performing a 3D CT data registration to a single interventional native fluoroscopic frame. Nasirudin et al 2015[8] have presented a Monte Carlo-based simulation of a SCT which has been equipped with a PCD. Yiu et al 2015[9] have proposed a new pixelbased, high-speed MV beamforming framework intended for performing synthetic aperture imaging for the formation of whole frames of adaptively apodized images at real-time throughputs. The proposed framework is primarily centered upon parallel computing principles. Strakos et al 2015[10] have presented the speedup of the k-means algorithm which is used for image segmentation. The authors have achieved this speedup with the help of effective parallelization using MIC architecture Intel Xeon Phi coprocessor. The authors used segmentation of CT images of the abdominal body part for demonstrating parallel capabilities of k-means algorithm. Kulak et al 2015[11] have developed the fuzzy axiomatic design also having the RFAD(risk factors) approach. The authors are the first to use this approach in comparing multi attributes of medical imaging systems. Hofmann et al 2014[12] have examined the Xeon Phi for its appropriateness in running the FDK algorithm. XeonPhi is centered upon Intel's MIC(Many Integrated Cores) architecture. FDK is the most frequently used algorithm for performing the 3D image reconstruction in the field of cone-beam computed tomography. Karonis et al 2013[13] have proposed the use of distributed and hardware-accelerated computing methods for achieving fast image reconstruction using pCT(proton computed tomography). Steuwer et al 2013[14] have presented SkelCL. SkelCL is a high-level programming model for the computing systems which are having multiple GPUs. The authors have implemented it as a library on top of OpenCL. Zhu et al 2012[15] have presented the implementation of a deconvolution algorithm for brain perfusion quantification on GPGPU (General Purpose Graphics Processor Units). The authors have made use of the CUDA programming model for the implementation. The authors have also evaluated the performance gains of such algorithms using GPUs and presenting both sequential as well as parallel implementations of those algorithms. Heckel et al 2011[16] have presented an interactive segmentation method for 3D medical images which is used for reconstruction of an object's surface by making use of implicit, smooth and energy-minimizing functions. Benquassmi et al 2011[17] have presented the parallelization of Katsevich CT image reconstruction method by making use of OpenMP. Then the authors have compared the results obtained with the CUDA results on 3 different GPGPUs. Fluck et al 2011[18] have presented a survey giving an overview of GPU accelerated image registration. The authors have addressed have surveyed programming models as well as interfaces, thus analyzing the different approaches for programming on GPU. Crane at al 2006[19] have proposed the integration of MR (Magnetic Resonance) scanners with HPC (high-performance computing) grid. The authors' objective has been to improve patient care by enabling near real-time, computationally intensive medical image processing, directly at an MR scanner. The authors have also described a graphical software tool, developed for running on the MR scanners. Warfield et al 1998[20] have developed an automatic registration algorithm to align the medical imaging data. The proposed algorithm works by measuring alignment by comparing dense feature sets (tissue labels).

\section{COMPARISON TABLE}

\begin{tabular}{|l|l|l|l|l|l|l|}
\hline \multirow{2}{*}{ Author Name } & Year & Paper Title & Technique Used & \multicolumn{3}{|l|}{ Parameters } \\
\cline { 3 - 7 } & & & & \multicolumn{2}{|l|}{$\begin{array}{l}\text { Parallelization } \\
\text { Couply } \\
\text { Distributed } \\
\text { Computing }\end{array}$} \\
\hline Doel et al [1] & 2017 & $\begin{array}{l}\text { GIFT-Cloud: A data } \\
\text { sharing and collaboration } \\
\text { platform for medical } \\
\text { imaging research[1] }\end{array}$ & $\begin{array}{l}\text { Cross-platform } \\
\text { technology used }\end{array}$ & $\mathbf{x}$ & $\checkmark$ & $\mathbf{x}$ \\
\hline Mingliang et al [2] & 2016 & $\begin{array}{l}\text { Medical image denoising } \\
\text { by parallel non-local } \\
\text { means[2] }\end{array}$ & $\begin{array}{l}\text { Improved version } \\
\text { of NLM } \\
\text { algorithm }\end{array}$ & $\checkmark$ & $\checkmark$ & $\mathbf{x}$ \\
\hline Guo et al [3] & 2016 & $\begin{array}{l}\text { Improved iterative image } \\
\text { reconstruction algorithm } \\
\text { for the exterior problem } \\
\text { of computed } \\
\text { tomography[3] }\end{array}$ & $\begin{array}{l}\text { Improved } \\
\text { iterative } \\
\text { algorithm } \\
\text { combining TVM } \\
\text { and RSF }\end{array}$ & $\mathbf{x}$ & $\checkmark$ & $\mathbf{x}$ \\
\hline Nguyen et al [4] & 2016 & $\begin{array}{l}\text { Medical image denoising } \\
\text { via optimal } \\
\text { implementation of non- } \\
\text { local means on hybrid } \\
\text { parallel architecture[4] }\end{array}$ & $\begin{array}{l}\text { Computation of } \\
\text { filters } \\
\text { coefficients }\end{array}$ & $\checkmark$ & $\checkmark$ & $\mathbf{x}$ \\
\hline
\end{tabular}




\begin{tabular}{|c|c|c|c|c|c|c|}
\hline Feng et al [6] & 2016 & $\begin{array}{l}\text { A Hybrid Parallel } \\
\text { Delaunay Image-to-Mesh } \\
\text { Conversion Algorithm } \\
\text { Scalable on Distributed- } \\
\text { Memory Clusters[6] }\end{array}$ & $\begin{array}{l}\text { 3-Dl hybrid } \\
\text { MPI+Threads } \\
\text { parallel Delaunay } \\
\text { image-to-mesh } \\
\text { conversion } \\
\text { algorithm } \\
\end{array}$ & $x$ & $\checkmark$ & $x$ \\
\hline Yiu et al [9] & 2015 & $\begin{array}{l}\text { GPU-Based Minimum } \\
\text { Variance Beamformer for } \\
\text { Synthetic Aperture } \\
\text { Imaging of the Eye[9] }\end{array}$ & $\begin{array}{l}\text { Minimum } \\
\text { Variance } \\
\text { Beamforming on } \\
\text { GPUs }\end{array}$ & $\checkmark$ & $\checkmark$ & $x$ \\
\hline Strakos et al [10] & 2015 & $\begin{array}{l}\text { Parallelization of the } \\
\text { image segmentation } \\
\text { algorithm for Intel Xeon } \\
\text { Phi with application in } \\
\text { medical imaging[10] }\end{array}$ & $\begin{array}{l}\text { k-means } \\
\text { algorithm }\end{array}$ & $x$ & $\checkmark$ & $x$ \\
\hline Hofmann et al [12] & 2014 & $\begin{array}{l}\text { Performance Engineering } \\
\text { for a Medical Imaging } \\
\text { Application on the Intel } \\
\text { Xeon Phi Accelerator[12] }\end{array}$ & $\begin{array}{l}\text { FDK algorithm } \\
\text { on XeonPhi }\end{array}$ & $\checkmark$ & $x$ & $x$ \\
\hline Karonis et al [13] & 2013 & $\begin{array}{l}\text { Distributed and hardware } \\
\text { accelerated computing } \\
\text { for clinical medical } \\
\text { Imaging using proton } \\
\text { computed tomography } \\
\text { (pCT)[13] }\end{array}$ & $\begin{array}{l}\text { Combining MPI } \\
\text { with GPUs }\end{array}$ & $\checkmark$ & $\checkmark$ & $x$ \\
\hline Steuwer et al [14] & 2013 & $\begin{array}{l}\text { High-level programming } \\
\text { for medical imaging on } \\
\text { multi-GPU systems using } \\
\text { the SkelCL library[14] }\end{array}$ & $\begin{array}{l}\text { SkelCL } \\
\text { implemented as a } \\
\text { library on top of } \\
\text { OpenCL }\end{array}$ & $\checkmark$ & $\checkmark$ & $x$ \\
\hline Zhu et al [15] & 2012 & $\begin{array}{l}\text { Parallel perfusion } \\
\text { imaging processing using } \\
\text { GPGPU [15] }\end{array}$ & $\begin{array}{l}\text { Deconvultion } \\
\text { algorithm } \\
\text { implemented on } \\
\text { GPGPU }\end{array}$ & $\checkmark$ & $\checkmark$ & $x$ \\
\hline Heckel et al [16] & 2011 & $\begin{array}{l}\text { Interactive 3D medical } \\
\text { image segmentation with } \\
\text { energy-minimizing } \\
\text { implicit functions[16] }\end{array}$ & $\begin{array}{l}\text { Interactive } \\
\text { segmentation } \\
\text { method having } \\
\text { energy - } \\
\text { minimizing } \\
\text { functions }\end{array}$ & $\checkmark$ & $x$ & $x$ \\
\hline $\begin{array}{l}\text { Benquassmi et al } \\
\text { [17] }\end{array}$ & 2011 & $\begin{array}{l}\text { Parallelization of } \\
\text { Katsevich CT image } \\
\text { reconstruction algorithm } \\
\text { on generic multi-core } \\
\text { processors and } \\
\text { GPGPU.[17] }\end{array}$ & $\begin{array}{l}\text { Katsevich CT } \\
\text { image } \\
\text { reconstruction } \\
\text { method }\end{array}$ & $\checkmark$ & $\checkmark$ & $x$ \\
\hline Crane et al [19] & 2006 & $\begin{array}{l}\text { Grid enabled magnetic } \\
\text { resonance scanners for } \\
\text { near real-time medical } \\
\text { image processing[19] }\end{array}$ & $\begin{array}{l}\text { Combining MR } \\
\text { scanners with } \\
\text { HPC grid }\end{array}$ & $x$ & $\checkmark$ & $x$ \\
\hline Warfield et al [20] & 1998 & $\begin{array}{l}\text { A high performance } \\
\text { computing approach to } \\
\text { the registration of } \\
\text { medical imaging data[20] }\end{array}$ & $\begin{array}{l}\text { automatic } \\
\text { registration } \\
\text { algorithm for } \\
\text { aligning medical } \\
\text { imaging data } \\
\end{array}$ & $x$ & $\checkmark$ & $x$ \\
\hline
\end{tabular}

\section{CONCLUSION}

Most of the Medical Imaging techniques need to be parallelized and the state-of-art techniques either require High Performance Computing (HPC) or GPUs for their processing. Implementing different techniques by making use of HPCs or GPUs result in increased cost of hardware. In this paper, the comparison of various approaches and algorithms used in medical imaging has been done. The comparison is done on the basis of whether they are implemented with the help of GPU or a parallelization based technique has been used. In the comparison, it is seen that, the state-of-art techniques are not effective for the distributed loosely coupled systems. This issue has not been considered by the existing literature so far. In near future, we will propose the implementation of k-means algorithm for computed tomography image segmentation on a framework consisting of distributed loosely coupled systems. For this implementation, the available hardware will be made use of and hence the hardware cost will be reduced manifold. 


\section{REFERENCES}

[1] Doel, Tom, Dzhoshkun I. Shakir, Rosalind Pratt, Michael Aertsen, James Moggridge, Erwin Bellon, Anna L. David, Jan Deprest, Tom Vercauteren, and Sébastien Ourselin: GIFT-Cloud: A data sharing and collaboration platform for medical imaging research. Computer methods and programs in biomedicine 139,pp.181-190 (2017)

[2] Mingliang, Xu, Lv Pei, Li Mingyuan, Fang Hao, Zhao Hongling, Zhou Bing, Lin Yusong, and Zhou Liwei: Medical image denoising by parallel non-local means. Neurocomputing 195,pp. 117-122 (2016)

[3] F Guo, Yumeng, and Li Zeng: Improved iterative image reconstruction algorithm for the exterior problem of computed tomography. Nuclear Instruments and Methods in Physics Research Section A: Accelerators, Spectrometers, Detectors and Associated Equipment (2016).

[4] Nguyen, Tuan-Anh, Amir Nakib, and Huy-Nam Nguyen: Medical image denoising via optimal implementation of non-local means on hybrid parallel architecture. Computer methods and programs in biomedicine 129, pp. 29-39 (2016).

[5] Bychkovsky, Brittany L., and Nancy U. Lin.: Imaging in the evaluation and follow-up of early and advanced breast cancer: When, why, and how often?. The Breast (2016).

[6] Feng, Daming, Andrey N. Chernikov, and Nikos P. Chrisochoides: A Hybrid Parallel Delaunay Image-to-Mesh Conversion Algorithm Scalable on Distributed-Memory Clusters. Procedia Engineering 163, pp. 59-71 (2016)

[7] Schwerter, Michael, Florian Lietzmann, and Lothar R. Schad: A novel approach for a 2D/3D image registration routine for medical tool navigation in minimally invasive vascular interventions. Zeitschrift für Medizinische Physik (2016).

[8] Nasirudin, Radin A., Petar Penchev, Kai Mei, Ernst J. Rummeny, Martin Fiebich, and Peter B. Noël: A Monte Carlo software bench for simulation of spectral k-edge CT imaging: Initial results. Physica Medica 31, no. 4, pp. 398-405 (2015)

[9] Yiu, Billy YS, and C. H. Alfred: GPU-Based Minimum Variance Beamformer for Synthetic Aperture Imaging of the Eye. Ultrasound in medicine \& biology 41, no. 3, pp. 871-883 (2015)

[10] Strakos, P., M. Jaros, T. Karasek, L. Riha, M. Jarosova, T. Kozubek, P. Vavra, and T. Jonszta: Parallelization of the image segmentation algorithm for Intel Xeon Phi with application in medical imaging. In 4th International Conference on Parallel, Distributed, Grid and Cloud Computing for Engineering. Civil-Comp Press, Stirlingshire(2015)

[11] Kulak, Osman, Hacer Guner Goren, and Aliye Ayca Supciller: A new multi criteria decision making approach for medical imaging systems considering risk factors. Applied Soft Computing 35, pp. 931-941 (2015)

[12] Hofmann, Johannes, Jan Treibig, Georg Hager, and Gerhard Wellein: Performance engineering for a medical imaging application on the Intel Xeon Phi accelerator. In Architecture of Computing Systems (ARCS), 2014 27th International Conference on, pp. 1-8. VDE (2014)

[13] Karonis, Nicholas T., Kirk L. Duffin, Caesar E. Ordoñez, Bela Erdelyi, Thomas D. Uram, Eric C. Olson, George Coutrakon, and Michael E. Papka: Distributed and hardware accelerated computing for clinical medical imaging using proton computed tomography (pCT). Journal of Parallel and Distributed Computing 73, no. 12, pp. 1605-1612 (2013).

[14] Steuwer, Michel, and Sergei Gorlatch: High-level programming for medical imaging on multi-GPU systems using the SkelCL library. Procedia Computer Science 18, pp. 749-758 (2013)

[15] Zhu, Fan, David Rodriguez Gonzalez, Trevor Carpenter, Malcolm Atkinson, and Joanna Wardlaw. Parallel perfusion imaging processing using GPGPU. Computer methods and programs in biomedicine 108, no. 3, pp. 1012-1021(2012)

[16] Heckel, Frank, Olaf Konrad, Horst Karl Hahn, and Heinz-Otto Peitgen: Interactive 3D medical image segmentation with energy-minimizing implicit functions. Computers \& Graphics 35, no. 2, pp. 275-287 (2011)

[17] Benquassmi, A., E. Fontaine, and H. H. S. Lee: Parallelization of Katsevich CT image reconstruction algorithm on generic multi-core processors and GPGPU. GPU Computing Gems 2, pp. 659-677 (2011).

[18] Fluck, O., Christoph Vetter, Wolfgang Wein, Ali Kamen, Bernhard Preim, and Rüdiger Westermann: A survey of medical image registration on graphics hardware. Computer methods and programs in biomedicine 104, no. 3, pp . e45-e57 (2011)

[19] Hy Crane, Jason C., Forrest W. Crawford, and Sarah J. Nelson: Grid enabled magnetic resonance scanners for near real-time medical image processing. Journal of Parallel and Distributed Computing 66, no. 12, pp. 1524-1533 (2006)

[20] Warfield, Simon K., Ferenc A. Jolesz, and Ron Kikinis: A high performance computing approach to the registration of medical imaging data. Parallel Computing 24, no. 9, pp. 1345-1368 (1998) 